\title{
Resozialisierung in der Lebenslaufperspektive
}

Eduard Matt

Resozialisierung (Reintegration, Wiedereingliederung) als Zielsetzung einer Vollzugspolitik unterliegt in letzter Zeit verstärkt der Kritik. Auf der einen Seite finden sich Tendenzen zu einer auf Härte und auf Strafverschärfung setzenden Kriminalpolitik. Die Herstellung der inneren Sicherheit wird betont, die resozialisierende Behandlung im Sinne der 3 Ts (therapy, treatment, training) tritt in den Hintergrund. Auf der anderen Seite wird deutlicher auf die Effektivität von Programmen, auf die nachweisbaren Erfolge von Resozialisierungsmaßnahmen insistiert. Ein weiterer, neuerer Strang der Argumentation nimmt eine lebenslauftheoretische Perspektive ein und läßt sich unter dem Begriff der ,Übergänge' fassen. Diese erst im Anfang sich befindende Diskussion um Übergänge in der Kriminologie soll im Folgenden in ihren Grundannahmen und Konsequenzen dargelegt werden, als geeignet, der Theorie und der Umsetzung des Konzeptes der Resozialisierung (wieder) neues Gewicht zu verleihen.

\section{Übergänge - Verfestigung und Ausstieg aus kriminellen Karrieren}

$\mathrm{M}$ it dem Konzept der Übergänge gelangt eine lebenslauftheoretische Perspektive in die soziologisch orientierte kriminologische Theorie und Forschung (Oberwittler, Karstedt (Hrsg.) 2004). Analysiert werden die Wege in die Straffälligkeit, die Dynamiken der Verfestigung (Persistence) und insbesondere die Formen des Ausstiegs aus ,kriminellen Karrieren (Desistance). Zur Analyse der Rolle und Bedeutung der Straffälligkeit im Lebenslauf werden die Themen der Strategien der beruflichen und sozialen Wiedereingliederung, der Reintegration, der Entlassungsvorbereitung sowie allgemeinerer/kriminalpolitischer Art, die Frage des Rückfalls relevant. Die unterschiedlichen Themen und Praktiken verweisen hierbei aber u.U. auf eine gemeinsame Zielsetzung.

Unter dem Konzept der Übergänge gelangen nicht so sehr die episodalen Täter, sondern die Wiederholungstäter, die wiederholt in Haft Einsitzenden, die Mehrfach- und Intensivtäter in den Fokus der Analyse. Die folgenden Ausführungen haben hierbei vor allem die Strafgefangenen im Blick. Für diese läßt sich aufzeigen, dass bei den Meisten neben der Straffälligkeit Mehrfachbenachteiligungen vorliegen (Drogen, Schulden, auffälliges soziales Verhalten, Gewaltbereitschaft, mangelnde schulische und berufliche Qualifikation, Langzeitarbeitslosigkeit, desolate Familienverhältnisse, Obdachlosigkeit, lange Sozialhilfekarrieren, mangelnde soziale Kompetenzen, traumatische Erfahrungen u.v.m.). Wir haben es mit Verlaufsformen zu tun, bei der anhaltend straffälliges Verhalten im Lebensverlauf erfolgt. Mit weiteren hierdurch entstehenden Problemlagen müssen sich die Personen auseinandersetzen: Stigma Vorbestraft-Sein, Finanzen, Identität, Probleme der Aufrechterhaltung persönlicher und sozialer Beziehungen.

Die aufgrund der Straffälligkeit entstehenden und entstandenen Problematiken für die Betroffenen sind kaum bekannt. Gerade über ihre Erwartungen, Befürchtungen und Bedarfe während der Haft sowie insbesondere für die Zeit `draußen $>$ im Rahmen der Entlassungsvorbereitung, ebenso wie ihre Erwartungen und Erfahrungen mit den Institutionen der Nachsorge (Bewährungshilfe, Berufshilfe, Freie Straffälligenhilfe, Sozialamt, Arbeitsamt u.v.m.) wissen wir nur sehr wenig. Welchen Problem- und Konfliktlagen sind sie nach der Haftentlassung ausgesetzt, welche Anforderungen werden an sie gestellt? Und wieso scheitern sie so oft an der Bewältigung dieser Situation?

\section{Resozialisierung, Sicherheit und Strafvollzug}

Gerade für den Strafvollzug ist die Perspektive der Resozialisierung von zentraler Bedeutung: Schließlich ist gefordert, dass die Insassen nicht schlechter herauskommen als sie hineingekommen sind (»Schädlichen Folgen des Freiheitsentzuges ist entgegenzuwirken« StVollzG § 3 II). Doch die Situation Haft kann alles andere als nicht schädigend sein: Man denke an die unterschiedlichen Formen der Haftanpassung, an Prisonierung, an Gewalthandlungen zwischen In- sassen, also allgemein an die schädlichen Einflüsse der Haftsituation auf den Gefangenen (vgl. Kury, Brandenstein 2002). Diesen gilt es ebenso $\mathrm{zu}$ begegnen. Eine entsprechende Ausgestaltung des Vollzugs ist und bleibt notwendig, eine Strategie des Wegschließens greift zu kurz. Der Anstieg der Strafgefangenenpopulation von 44278 im Jahre 1994 auf 60742 im Jahre 2002 zeigt die Aufgabe, vor der der Vollzug zur Zeit steht. Er bringt aber auch ein weiteres, weniger beachtetes Problem mit sich: Sind 199467545 Gefangene aus der Haft entlassen worden, so sind es 2002 bereits $74244 .{ }^{1}$ M.a.W.: Über kurz oder lang wird jeder Inhaftierte wieder entlassen (nur sehr wenige werden es nicht). Und es bleibt die Frage: Wie verhalten sie sich danach? Eine ausschließliche Inhaftierung, ohne Bildung und Beschäftigung, ohne qualifiziert gewährte Lockerungen, Ausgang und Urlaub, würde die Insassen völlig unvorbereitet in das Leben draußen entlassen - mit allen Konsequenzen. Notwendig ist die Gestaltung des Übergangs, seien es entsprechende Vorbereitungen, sei es eine Nachbetreuung, und dies in Kooperation der unterschiedlichen Institutionen. So gesehen ist und bleibt die Strategie der Resozialisierung notwendig für die Wiedereingewöhnung in das normale Leben. Resozialisierung dient zugleich dem Schutz der Allgemeinheit, der Sicherheit! Gegen eine populistische Forderung von Sicherheit statt Resozialisierung fordert die aufgeklärte Kriminalpolitik: Sicherheit durch Resozialisierung.

Statt von Resozialisierung sollte besser von (sozialer) Reintegration gesprochen werden. Der Begriff der Reintegration ist weniger auf defizitäre Persönlichkeitsmerkmale bezogen als auf defizitä- 
re soziale Einbindungen jeglicher Art (Arbeit, Verhalten, Kompetenzen). Mit ihm wird deutlicher auf die Situation von Straffälligen Bezug genommen und es ist von vornherein klar, dass es nicht zu einer Verfestigung krimineller Karrieren kommen soll. Ziel ist die Wiedereingliederung in die Gesellschaft. Resozialisierung ist vielleicht nicht bei allen Straffälligen notwendig, aber Formen der Reintegration sind es. Hier gewinnen die Maßnahmen und Programme sowie die Entlassungsvorbereitung in der JVA ihren zentralen Stellenwert. Notwendig ist der Austausch zwischen Institutionen der Straffälligkeit und der Gemeinschaft. Nur eine gemeinsame Politik aller beteiligten Institutionen kann die Perspektive der Reintegration zu einer erfolgversprechenden Zielsetzung der Reaktion auf Straffälligkeit machen. Betroffen bei der Frage der Resozialisierung/Reintegration ist zugleich die nach der Motivation der Straffälligen, sich auf die Wiedereingliederung in die Gesellschaft vorzubereiten. Erfahrungen im Bereich der (Neu-)Implementierung schulischer und beruflicher Maßnahmen in einer JVA zur Reintegration von Straffälligen ${ }^{2}$ in den Arbeitsmarkt machen diese Problematik besonders deutlich. Erst bei Entstehung von Motivation und positivem Selbstbild durch erste Erfolge in Maßnahmen zur schulischen und beruflichen Bildung erfolgt eine aktive und erfolgreiche Teilnahme. Die Teilnehmer aus ihrer Lethargie $\mathrm{zu}$ bringen ist mit ein wesentliches Element der Maßnahmen. ${ }^{3}$

\section{Verortungen des Ansatzes}

Die Diskussion zur Übergangsproblematik liegt erst in den Anfängen. Das Wissen speist sich hierbei aus unterschiedlichen Quellen: aus der Rückfallforschung, aus den wenigen Untersuchungen zum Verlauf und Abbruch langer krimineller Karrieren, aus Längsschnittstudien (z.B. Schumann 2003a, b), aus den Erfahrungen aus dem Bereich der Entlassungsvorbereitung in den Justizvollzugsanstalten, aus den neuen Ansätzen zur schulischen und beruflichen Resozialisierung. Zu nennen sind ferner die Arbeiten aus dem Bereich der Wirkungsforschung in der Folge des auch im deutschsprachigen Raum rezipierten ShermanReports über die Effektivität von Maßnahmen (Bannenberg, Rössner 2003). Im englischsprachigen Bereich finden sich unter dem Begriff der Wiedereingliederung (,re-entry') diverse Arbeiten zur Thematik, gerade unter dem Aspekt der Effektivität in der Perspektive einer auf Evidenz basierenden Politik, sowohl für den Bereich der Gefängnisse als auch insbesondere für die Bewährungshilfe (Farrall 2002, Seiter 2003).

\section{Die Rückfallforschung}

Die Rückfalluntersuchungen nutzen als Datenquelle meist die Eintragungen im Bundeszentralregister (BZR) (siehe zuletzt Jehle et al. 2003).
So zeigt gerade die Rückfallforschung besonders schlechte Zahlen für Strafgefangene, es finden sich deutlich hohe Quoten. Wir erfahren hier viel darüber, dass und wie oft ein Rückfall erfolgt, aber über das wie und warum erfahren wir nichts. Was fehlt, ist die Analyse der situativen bzw. sozialen Dynamik der Situation: Welche Elemente führen zum Rückfall, wie sieht die soziale und biographische Einbettung aus? Der Vorgang wird eher unter dem Aspekt des Outputs erforscht, in der Regel ausschließlich anhand der Eintragungen im BZR. Das Wissen um die damit verbundenen Prozesse (des Rückfalls, der Desistance) wiederum ist geringer. Gerade bei den BZR-Daten fehlt jeglicher Verweis auf andere Teilbereiche des Lebens, auf Strukturen des Lebenslaufes als strukturelle Merkmale. Die Erfahrungsdimension, eine Kontextualisierung des Geschehens ist stärker gefordert. Gleichwohl ist der Rückfall das wichtigste Erfolgskriterium in der kriminalpolitischen Diskussion, es dient zur Evaluation von therapeutischen, qualifizierenden u.a. Interventionen - wenn auch mit dem Ergebnis, dass diese oftmals schlecht abschneiden. Neben der meist viel zu kurzen Zeitperspektive, einem kurzfristigen Zweckdenken dürfte der Grund in der eher punktuellen Betrachtungsweise liegen: sie ist nur auf die Intervention bezogen, nicht auf die Lebenslage der Betroffenen. Aber es finden sich inzwischen differenziertere Einschätzungen.

\section{Arbeit und Wiedereingliederung}

In den letzten Jahren ist wieder der zentrale Aspekt von Arbeit und Ausbildung für die Resozialisierung/Reintegration Straffälliger verstärkt betont worden. Durch Arbeitstraining, schulische und berufliche Ausbildung und Qualifizierungsmaßnahmen sollen (erste) berufliche Qualifikationen erworben werden. Insbesondere Arbeitstugenden, soziale Kompetenzen und Schlüsselqualifikationen werden trainiert und auf diese Weise die Möglichkeiten des Straffälligen nach Entlassung auf dem Arbeitsmarkt verbessert. ${ }^{4}$ Der Stand der Diskussion über die notwendige Vermittlung beruflicher Qualifikationen für Straffällige sagt ferner übereinstimmend, dass zugleich eine verstärkte Beschäftigung mit der Zeit nach der Haft erfolgen muß. Entscheidende Bedeutung für die Reintegration und die Vermeidung von Rückfälligkeit hat eine gelungene Vermittlung in Ausbildung oder Arbeit unmittelbar nach der Entlassung. Für die Situation Vollzug zeigt sich sehr deutlich, wie wichtig eine intensive systematische langfristige Betreuung ist. Als Erfolg versprechend erweist sich eine systematische Betreuung und Nachbetreuung im Übergang zur Wiedereingliederung.

Studien zeigen deutlich die delinquenzsteigernde Bedeutung langfristiger Arbeitslosigkeit. Bei einer systematischen Betreuung durch konsequente Qualifizierung in Haft mit anschließender Einbin- dung in weitere Arbeit oder Ausbildung nach der Haftentlassung wird eine deutliche Rückfallminderung erreicht (Wirth 1998; Hammerschick 2000). Für ein weiteres Halten in Arbeitslosigkeit und Delinquenz ist ebenso die Reaktionsform der Justiz von Bedeutung: die mangelnde berufliche Integration wird als Kriterium bei der Entscheidung zur Verhängung einer unbedingten Freiheitsstrafe genommen (Schumann 2003a). ${ }^{5}$ Resozialisierung führt aber nicht zwingend $\mathrm{zu}$ Legalverhalten, beruflich Etablierte begehen ebenso Straftaten (meist im Bagatellbereich). Insofern ist das Rückfallrisiko kein guter Indikator für den Erfolg von Resozialisierung. Doch eine Integration in die Gesellschaft wird wieder ermöglicht. $\mathrm{Zu}$ gleich verweist Schumann zurecht darauf, dass Resozialisierungsmaßnahmen nicht aufgezwungen werden dürfen. Die Ausgrenzung der Personen ist meist Ergebnis einer langen Entwicklung mit mehrfachen Belastungen, die durch kurze Maßnahmen nicht auszugleichen ist (ebd.).

\section{Längsschnittstudien}

Eine weitere wichtige Quelle von Erkenntnissen zur Problemstellung sind kriminologische Längsschnittstudien. Untersucht werden zwar im Grunde alle statusbezogenen Faktoren, doch keiner hat sich eindeutig als determinierend erwiesen: Arbeit, Aufgabe von Sucht, Aufnahme einer stabilen Partnerschaft, Verlassen von Cliquen und Milieus u.a. (Laub, Sampson 2001). Gefunden wurden bisher aber keine kausalen Mechanismen des Ausstiegs, nur Wahrscheinlichkeiten, unterschiedliche Risikolagen, probabilistische Aussagen. Über die genaue Wirkungsweise, über das ,Wie es vor sich geht und,Warum es geschieht' wissen wir wenig. Insofern ist es durchaus noch eher unklar, wie das Ziel der Konformität $\mathrm{zu}$ erreichen ist. Zugleich weisen einige Studien aber gerade die Möglichkeiten des Aufhörens, des Ausstiegs aus Delinquenz auf; er kann schleichend oder plötzlich erfolgen (empirisch meist schleichend). Die Studien zeigen auf, dass selbst Personen mit jahrzehntelangen >kriminellen Karrieren ‘ der Reintegration zugänglich sind (Stelly, Thomas, Kerner 2003). Doch über den Weg in die Freiheit wissen wir nicht genügend - außer dass sie zurückkommen.

Die Längsschnittstudien, rezipiert in der Entwicklungspsychopathologie (Lösel, Bliesener 2003; Farrington Coid (Hrsg.) 2003), verdeutlichen zugleich, dass oftmals bereits vor Eintritt des Strafmündigkeitsalters von 14 Jahren massive Problemlagen und Auffälligkeiten vorliegen. Entwicklungswege von Verhaltensauffälligkeiten in früher Kindheit, von traumatischen Erfahrungen und gestörten familialen und sozialen Kontexten, die Akkumulation ausschließlich negativer Selektionserfahrungen hin zu späterer Delinquenz und Straffälligkeit lassen sich dergestalt nachvollziehen. Dem zeitlichen Verlauf der Abwärtsspirale wird im Sinne des ıwechselseiti- 
gen Aufschauklungsprozesses (Quensel 1970) Rechnung getragen. Die Entwicklung von den ersten Eigenarten und Auffälligkeiten hin zu einer negativen Karriere bis hin zum Gefängnisaufenthalt läßt sich als ein Wechselprozess von (sozial) auffälligen Verhalten und sozialen Reaktionen begreifen. Fehlende soziale Unterstützung, inadäquate Hilfsangebote, schwierige familiale und soziale Situationen sowie im späteren Verlauf u.U. auch justitielle Reaktionen, verstärken den Prozess. Weiterhin verdeutlichen die Studien, dass wir nicht nur auf Risikolagen und Risikofaktoren fokussieren dürfen, sondern ebenso die protektiven Faktoren (vor allem: die Qualität sozialer Beziehungen, sei es in Familie, Schule, auf der Arbeit, zu Freunden u.a. sowie die gute Integration in die Arbeitswelt) in die Analyse aufnehmen sollten. Erst dies ermöglicht u.U. $\mathrm{zu}$ verstehen, warum trotz oftmals vergleichbaren Risikolagen nur ein Teil der Betroffenen straffällig wird. Ein weiterer Aspekt ist in der kriminologischen Längsschnittforschung in den letzten Jahren hierbei verstärkt untersucht worden: die Formen und Wege des Ausstiegs (Desistance).

\section{Was leistet eine Lebenslaufperspektive?}

Gerade die Überlegungen zum Aussieg aus einer >kriminellen Karriere haben zu einer stärker auf den Lebenslauf bezogene Betrachtungsweise beigetragen. Ausstieg wird als längerer Prozess betrachtet, nicht als Ereignis. Die Dynamik des Ausstiegs einschließlich des Zusammenhang mit den Veränderungen der Rollen der Person und des sozialen Kontextes wird untersucht. $\mathrm{Zu}$ nennen ist hier insbesondere die Arbeit von Shadd Maruna (2000). Er versucht, die Perspektiven der Betroffenen in der Analyse aufzunehmen. Desistance wird im Zusammenhang gesehen mit Veränderungen des Selbst (self-change). Fragen des Selbstbildes und der Selbst-Identität - und im Prozess des Ausstiegs entsprechende Reorganisationen- kommen ins Blickfeld. Dergestalt bringt Maruna die Personen zurück in die Analyse. Die Individuen werden als Agenten ihrer eigenen Veränderung gesehen, und Veränderung wird als Prozess konzeptualisiert, als Ergebnis der Auseinandersetzung des Handelnden mit den sozialstrukturellen und institutionellen Faktoren. Die Eigenaktivität der Person gewinnt in den Analysen an Bedeutung.

So weist Maruna aus einer Lebenslaufperspektive darauf hin, dass die einzelnen Programme, therapeutische Interventionen und andere Maßnahmen für sich genommen eher einen begrenzten Erfolg aufweisen. Gerade der Vergleich der Leistungen von Programmteilnehmern mit der normalen Straffälligenpopulation führte zu dem Ergebnis, dass der Effekt der formalen, professionellen Interventionen (Therapie, Qualifizierungen u.a.) auf den Rückfall überhaupt nicht einschätzbar ist: in der normalen Population gab es ebenso viele sspontane Remissionen`. Die Aus- stiegsformen aus Delinquenz waren vergleichbar. Die Mechanismen entstehen in Auseinandersetzung mit anderen, seien es Professionelle, seien es Laien und Bezugspersonen (Maruna et al. 2004, S. 12). So gesehen sind ebenso die NichtTeilnehmer durchaus sInterventionen ausgesetzt, in Form von Unterstützung, Betreuung und Kritik durch Freunde, Partner, Familie. Und diesen Einflüssen sind sie in der Regel bedeutend länger ausgesetzt als formalen Interventionen, und es sind Personen, die ihnen in der Regel etwas bedeuten.

In den Blick gerät etwas, das man Selbstmanagement nennen könnte: die Fähigkeit, sein eigenes Leben selbst $\mathrm{zu}$ organisieren. Voraussetzung und notwendige Bedingung für einen Ausstieg ist so gesehen erst einmal und vor allem die eigene Motivation: man muß es selber wollen. Die Zielsetzung einer Veränderung des Selbst und der Lebensführung muß der Betroffene selbst angehen. Diese Rebiographisierung (self-reform) ist zugleich gebunden an die soziale Integration $(\mathrm{Ar}-$ beit, Beziehungen u.a.), an die Situationen, in denen man lebt. Neben den Veränderungen von Persönlichkeit und Selbst-Konzept gehört ebenso die Übernahme von Verantwortung für sich selbst und sein Leben. Als ein weiteres Element kann hinzukommen, dass die Person einsieht, anderen (oder gar der Gesellschaft) geschadet $\mathrm{zu}$ haben, und dies zukünftig vermeiden will, also anderen keinen Schaden mehr zufügen will, diesen gar beheben oder etwas zurückgeben will. Es wird ebenso zur Aufgabe, die lange Zeit der Delinquenz nicht als verlorene Zeit in seiner Biographie zu betrachten. Eine derartige Thematisierung der Übergänge führt zu einer dynamischen Betrachtungsweise des Werdegangs, des Verlaufs und der darin verwobenen unterschiedlichen Konstellationen von Delinquenz und Lebenslauf. Oder anders formuliert, es führt zu einer prozessualen Betrachtungsweise, nicht zu einer punktuellen. Als Ergebnis lassen sich unterschiedlich wirkende Faktoren finden, innen und außen, unterschiedlich wirkend im Zeitverlauf.

Die Konzeptualisierung richtet sich zugleich gegen eine spezifische Deutung des maturing out, gegen jene, die mit einer Strategie der Nicht-Reaktion, des `Nichts-Tuns`, des Abwartens verbunden ist. Dieser wird entgegengehalten, dass Anstöße notwendig sind, Erfahrungen gemacht und verarbeitet werden, Positionen verdeutlicht und Mißfallen kundgetan wird und werden muss. Das Tatunrecht ist zu verdeutlichen (die Formen sind zu diskutieren). Erst im Prozess eines derartigen >maturing-out< werden Erfahrungen und Anstöße zu einem neuen Selbstbild verarbeitet werden können. So gesehen gehören notwendigerweise zum maturing out entsprechende Lebenserfahrungen und -veränderungen.

Dem entsprechen die Ergebnisse der Tübinger Jungtäter-Vergleichsuntersuchung. Bei der Analyse der langjährigen Verläufe zeigte sich gerade als zentraler Faktor die sinnere Umorientierungen der Probanden<, der Weg des Ausstieges als ein langwieriger, mit Rückschlägen und Phasen der Unsicherheit. Wichtig ist hierbei, dass Veränderungen eintreten, weniger wichtig, in welchem Bereich.

»Die neueren Befunde aus der internationalen Forschung stimmen in verschiedenen Ländern im Grunde nach erstaunlich gut überein. Sie können tentativ dahin gehend zusammengefasst werden, dass es nicht so sehr darauf ankommt, an welcher Stelle oder in welchem Bereich die Veränderung einsetzt. Vielmehr kommt es darauf an, dass überhaupt eine neue Lage irgendwo eintritt oder hergestellt wird, und dass sie den Täter in einer sozusagen sensiblen Lebenssituation trifft. Wenn der gute Anfang gemacht ist, knüpft sich das weitere soziale Netz viel leichter, manchmal von außen aus betrachtet fast wie von selbst, neu und vergleichsweise oder sogar gleich ganz stabil« Kerner 2004, S. 43f..

Anregungen, Anstöße, Erfahrungen, aber auch Interventionen und andere Maßnahmen gewinnen dergestalt einen Effekt im Lebenslauf der Betroffenen, oftmals verzögert, in einem langen Prozess der Entwicklung und Veränderung. Insofern sind sie notwendig, selbst wenn ihr Erfolg auf den ersten Blick eher sehr gering ausfällt. Kerner spricht hier von einer kognitiven Resozialisierung ${ }_{<}(2004$, S. 52), die genau diese Neu-Organisation der Person, die Veränderung des eigenen Selbstbildes erfasst.

\section{Schlußfolgerungen}

In der lebenslaufbezogenen Perspektive wird der Gedanke, dass das Individuum sozial eingebettet ist, dass soziale, situative und biographische Merkmale für die Entwicklung des Individuums relevant sind, wieder hervorgehoben. Die soziale Situation und die biographische Entwicklung bilden den Rahmen, innerhalb dessen das Individuum sein Leben selbst aktiv gestaltet. Kriminelles Verhalten ist so gesehen unangemessenes Verhalten zur Lösung spezifischer biographischer Problemlagen. Aus der Lebenslauf-Perspektive ergibt sich die Notwendigkeit einer Stärkung des Individuums (Resozialisierung, Empowerment), sei es auf der individuellen Ebene (Konfliktlösungsstrategien, Kompetenzen u.a.), sei es auf der sozialen Ebene (berufliche Qualifikationen, soziales Umfeld u.a.) (Günther 2002, S. 127f.). Erst der Erwerb dieser Fertigkeiten, so der Gedanke der Resozialisierung/Reintegration, ermöglicht es den Straffälligen, ihr Leben selbst zu gestalten - und dies, so die Hoffnung, ohne Straffälligkeit.

Für eine Neukonzeptualisierung der Resozialisierung (Reintegration, Wiedereingliederung) lassen sich folglich einige Kriterien angeben: sie muß langfristig ausgelegt sein, als längerer Prozess aufgefasst werden, sie muß sich durch Ernst- 
haftigkeit, Nachhaltigkeit und Einzelfallorientierung auszeichnen. Aufgabe von Interventionen ist es, die Handlungsfähigkeit der Teilnehmer zu erhöhen. ${ }^{6}$ Erst durch die Übernahme von Verantwortung und durch die Aneignung entsprechender Kompetenzen wird es den Individuen möglich, ihr Leben selbst zu gestalten. Die notwendigen Fähigkeiten und Fertigkeiten für die Integration in die Gesellschaft müssen entwickelt werden. Die meisten Straffälligen verfügen über nur sehr geringe Ressourcen aller Art (materieller Art, bildungsmäßige Voraussetzungen, Dispositionen der Selbstkontrolle und Selbststeuerung, kulturelle Ressourcen, Konfliktlösungspotential, soziale Kompetenzen u.v.m.). Aufgabe der Resozialisierung/Reintegration ist die Verbesserung der Ressourcen. Erst dies ermöglicht den Betroffenen die Entwicklung und Wiedergewinnung von Handlungsspielräumen. Sie sind zugleich notwendig für das Eingehen von Beziehungen jeglicher Art. Die Einbindung in Beziehungen mit den dazugehörigen Verpflichtungen stellt eine positive und effektive Integration in die Gesellschaft dar. Seitens der Gemeinschaft bedeutet es, die Täter werden wieder aufgenommen, sie erhalten die Chance, sich als nützliches Mitglied zu bewähren. ${ }^{7}$ Erst damit kann der Übergang als gelungen angesehen werden. Mit der Zielsetzung der Reintegration wird zur Aufgabe einer aufgeklärten Kriminalpolitik die Förderung der Ermöglichung derartiger Übergänge und die Minimierung von diese hindernden Faktoren und Situationen. Oder in den Worten eines Strafgefangenen:

"... längere Zeit [des Einsitzens] ist nicht gut, ist nicht gut für uns, nicht gut für körperlich, für gesundheitlich, für menschlich. Also- Wir brauchen das- Sozialkontakte mit draußen. Das ist doch der große Fehler, was die [die Kriminalpolitik] machen ... die wollen uns gewinnen oder verlieren? Was wollen die von uns, was erwarten die von uns?«

Dr. Eduard Matt arbeitet als Soziologe beim »Bremer Verein zur Förderung der interdisziplinären Zusammenarbeit zwischen Wissenschaft, Praxis und Politik im Bereich sozialer Probleme " (BRESOP e.V.) und ist wissenschaftlicher Begleiter des Projektverbundes "Chance in Bremen.

\section{Literatur:}

Bannenberg, Britta; Dieter Rössner: Preventing Crime: What works, what doesn't, what's promising? - Der Sherman-Report und seine Bedeutung für die deutsche Kriminalprävention. In: Zeitschrift für Jugendkriminalrecht und Jugendhilfe 2003, S. 111-119

Bazemore, Gordon; Carsten Erbe: Reintegration and restorative justice: Towards a theory and practice of informal social control and support. In: Shadd Maruna, Russ Immarigeon (Hrsg.): After Crime and Punishment. Pathways to offender reintegration. Cullompton 2004, S. 27-56

Dünkel, Frieder: Resozialisierungsvollzug (erneut) auf dem Prüfstand. In: Jörg-Martin Jehle
(Hrsg.): Täterbehandlung und neue Sanktionsformen. Mönchengladbach 2000, S. 379-414

Dünkel, Frieder; Kristin Drenkhahn: Behandlung im Strafvollzug: von »nothing works « $z u$ »something works «. In: Mechthild Bereswill, Werner Greve (Hrsg.): Forschungsthema Strafvollzug. Baden-Baden 2001, S. 387-417

Farrington, David P.; Jeremy W. Coid (Hrsg.): Early Prevention of Adult Antisocial Behaviour. Cambridge 2003

Günther, Klaus: Zwischen Ermächtigung und Disziplinierung. Verantwortung im gegenwärtigen Kapitalismus. In: Axel Honneth (Hrsg.): Befreiung aus der Mündigkeit. Paradoxien des gegenwärtigen Kapitalismus. ffm 2002, S. 117-139

Hammerschick, Walter: Arbeitsmarktintegration Straffälliger - Was ist »Good practice«? In: Kriminologisches Journal 32, 2000, S. 56-65

Jehle, Jörg-Martin; Wolfgang Heinz; Peter Sutterer: Legalbewährung nach strafrechtlichen Sanktionen. Eine kommentierte Rückfallstatistik. hg. vom BMJ; Berlin 2003

Kerner, Hans-Jürgen: Freiheit und Unfreiheit. Zum Verlauf der Karrieren von Straftätern. In: Gerhard Rehn et al. (Hrsg.): Freiheit und Unfreiheit. Arbeit mit Straftätern innerhalb und außerhalb des Strafvollzuges. Herbolzheim 2004, S. 3-53

Kury, Helmut; Martin Brandenstein: Zur Viktimisierung (jugendlicher) Strafgefangener. In: ZfStrVo 51, 2002, S. 22-33

Laub, John, H.; Robert J. Sampson: Understanding desistance from crime. In: Crime and Justice 28 , 2001, S. 1-69

Lösel, Friedrich; Thomas Bliesener: Aggression und Delinquenz unter Jugendlichen. Untersuchungen von kognitiven und sozialen Bedingungen. Neuwied 2003

Maruna, Shadd: Making Good. How Ex-Convicts reform and rebuild their Lives. Washington 2000

Maruna, Shadd; Russ Immarigeon (Hrsg.): After Crime and Punishment. Pathways to Offender Reintegration. Cullompton 2004

Matt, Eduard: Verantwortung und (Fehl-)Verhalten. Für eine restorative justice. Münster 2002

Matt, Eduard: Der Projektverbund »Chance in Bremen: Konzeption und Praxis. Zeitschrift für Strafvollzug und Straffälligenhilfe 52, 2003, S. 81-88

Oberwittler, Dietrich; Susanne Karstedt (Hrsg.): Soziologie der Kriminalität. Wiesbaden 2004

Quensel, Stephan: Wie wird man kriminell? In: Kritische Justiz 3, 1970, S. 377-382

Schumann, Karl F. (Hrsg.): Berufsbildung, Jugend und Delinquenz. Bremer Längsschnittstudie zum Übergang von der Schule in den Beruf bei ehemaligen Hauptschülern. Bd. 1, Weinheim/ München 2003a

Schumann, Karl F. (Hrsg.): Delinquenz im Lebenslauf. Bremer Längsschnittstudie zum Übergang von der Schule in den Beruf bei ehemaligen Hauptschülern. Bd. 2, Weinheim/München 2003b

Seiter, Richard P.: Prisoner Reentry:What works, what does not, and what is promising. In: Crime and Delinquency 49, 2003, S. 360-388

Sherman, Lawrence et al.: Preventing crime: What works, what doesn't, what's promising. National Institute of Justice. July 1998 [online]

Stelly, Wolfgang; Jürgen Thomas; Hans-Jürgen Kerner: Verlaufsmuster und Wendepunkte in der Lebensgeschichte. Tübingen 2003 [online]

Wirth, Wolfgang: Prävention durch Wiedereingliederung in den Arbeitsmarkt: Cui bono? In: Gabriele Kawamura, Ursula Helms (Hrsg.) Straffälligenhilfe als Prävention? Freiburg 1998, S. $55-75$

\section{Fußnoten:}

1 Statistisches Bundesamt, Strafverfolgungsstatistik, Fachserie 10, Rechtspflege, Reihe 4.1, 4.2. 2 Das Projekt "Chance" hat eine Laufzeit vom 1.11.2000-31.12.2004 und wird gefördert vom Senator für Arbeit, Frauen, Gesundheit, Jugend und Soziales, der Europäischen Gemeinschaft (ESF) (Förderschwerpunkt 10: Qualifizierung im Strafvollzug), den Arbeitsämtern Bremen und Bremerhaven sowie dem Senator für Justiz. Projektträger ist JUDIT Bremen im Auftrag der JVA Bremen. Siehe ausführlich: Matt 2003; http://www.chance.uni-bremen.de.

$3 »$ Mit fortschreitender krimineller Karriere wächst aber auch die Gefahr der inneren Unfreiheit, d.h. der nach und nach dichter und schließlich scheinbar ausweglos werdenden Verstrickung in Lebensbezügen und objektiven wie subjektiven Determinanten, die der viel berufene Kreislauf von Verbrechen und Strafe mit sich bringt « Kerner 2004, S. 3.

4 Die vor fast 30 Jahren zur Einschätzung von Resozialisierungsmaßnahmen aufgekommene These des >nothing works< ist inzwischen differenziert worden. So haben Sherman et al. (1998) in ihrer Meta-Evaluation die unterschiedlichen Maßnahmen noch einmal ob ihrer Wirksamkeit (»what works, what doesn't work, what's promising, what we don't know«) eingeschätzt. Vgl. ebenso: Dünkel 2000; Dünkel, Drenkhahn 2001.

5 »Man kann also bilanzierend sagen: nicht die aktuelle Arbeitslosigkeit, wohl aber eine wiederkehrende, durch Sanktionierungsprozesse stabilisierte Arbeitslosigkeit erhöht durch die mit Akkumulation einhergehende Marginalisierung das Risiko zu weiterer Delinquenz ... langfristig berufliche Etablierung [erwies sich] als die Konformität fördernd ... Dagegen wurden erfolgreich Qualifizierte, die sich längerfristig nicht etablieren konnten, in weit höherem Maße delinquent als solche, denen die Etablierung gelungen war « Schumann 2003a, S. $248 \mathrm{f}$..

6 Gegen die spezifische Deutung von Resozialisierung als Behandlung ist ebenfalls zu argumentieren. Sie setzt eine passive Rolle der Straffälligen und eine aktive Rolle der Institutionen voraus. Gegen diese paternalistische Haltung wird die Forderung nach Übernahme von (Eigen-)Verantwortung für das Führen des eigenen Lebens als Zielsetzung gesetzt (als soziale Verantwortung, nicht als Selbstverantwortung im neoliberalen Sinne). So gesehen gilt es, die Straffälligen zu befähigen, ihre Handlungsspielräume zu erweitern (soziale Kompetenzen, schulische und berufliche Qualifikationen u.a.), um dergestalt eine neue Rolle in der Gemeinschaft einnehmen zu können. Der Straffällige wird als Subjekt aufgefasst, als Agent seines Lebens, nicht als Objekt der Einwirkung externer Instanzen, denen er unterworfen ist.

7 Mit der Betonung der Auseinandersetzung mit der Tat und insbesondere mit den Tatfolgen, Fragen der Wiedergutmachung sowie der Rolle der Gemeinschaft zeigen sich deutlich Querverbindungen zum Ansatz der restorative justice. Vgl. Bazemore, Erbe 2004; allg. Matt 2002. 